\title{
Joints dynamic identification and modeling based on FRFs data
}

\author{
Guanhua Dong, Wei $\mathrm{Wu}^{*}$, and Jianhui Zhou \\ China Ship Development and Design Center, PR China 430000
}

\begin{abstract}
The problem of joints dynamic identification and modeling is discussed in this paper. The theoretical dynamic model of joints is established by FRFs (frequency response functions) data, and formulas for identifying the joints dynamic properties is deduced. The equivalent value of dynamic stiffness is extracted by solving the inconsistent equation using the least square method. The experimental example is provided to validate the feasibility and accuracy of the proposed method, the predicted result showing good fitting with experimental results.
\end{abstract}

\section{Introduction}

Mechanical structures are generally composed of substructures which are integrated by joints (such as bolts, welds and glue). When the mechanical system vibrates, the energy is absorbed, stored and released by joints, which reflect the stiffness and damping of joints dynamic characteristic. Due to the complexity and variability of joints' dynamics properties, purely theoretical analysis or finite element modeling could not establish the dynamical model of joints $[1,2]$. The way to extract the joints' properties from measured data becomes one of the research hotspots in the related field.

The most suitable approach from the practical point of view is using frequency response function (FRF) data to identify the dynamic characteristic of joints, which can be measured directly. The first FRF method for the joint identification was probably mentioned by Okubo and Miyazaki [3], and developed by Tsai and Chou [4], Ren and Beards [5]. The model of joints was considered as a set of linear spring-damper elements in translational degree of freedom in above researches. Thereafter, the impact of rotational degree of freedom was investigated by Liu [6]. Instead of just a set of translational and rotational springs, the model of joints was considered as a coupled stiffness matrix in [7]. The identification formulas were deduced by Čelič, and the influence of the coordinate reduction on the identification of the joint dynamic properties was investigated in [8]. In above researches, there is a common problem: in the identification process, FRF data can't be measured completely, to solve this problem, the FRFs data of joints coordinates system is estimated by the measurable FRF or ignored. No matter in which way, few errors are mixed into the following identification formulas, and the influence of the errors is not ignorable at times.

The purpose of this paper is to discuss the problem of the joints dynamic identification

\footnotetext{
*Corresponding author: guanhua1296@qq.com
} 
and modeling based on FRFs data. The theoretical dynamic model of joints is established by FRFs (frequency response functions) data, and formulas for identifying the joints dynamic properties are deduced. The equivalent value of dynamic stiffness is extracted by solving the inconsistent equation using the least square method. The experimental example is provided to validate the feasibility and the accuracy of the proposed method, showing good fitting with experimental results.

\section{Theoretical model}

In this paper, the mechanical structure is divided into three systems: the first system is the substructure system consisting of substructures, the second system is the joint system comprised of joints, and the third system is the assembly system assembled by the substructure system and joint systems. Schematic diagram of theoretical model is shown in figure 1 .

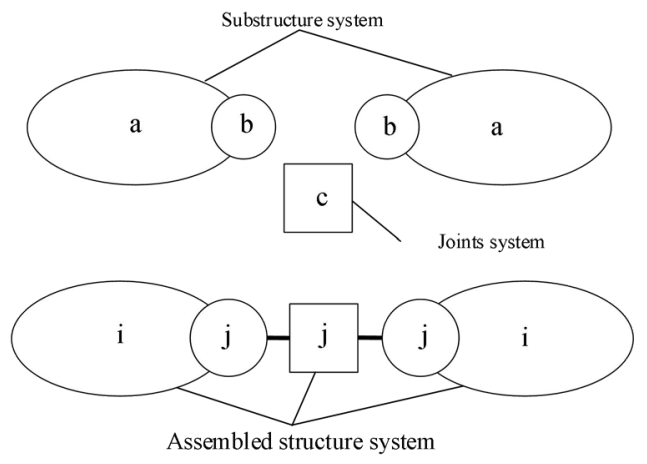

Fig. 1. Schematic diagram of theoretical model.

The input-output relationship of an assembled structure can be expressed as

$$
\left\{\begin{array}{l}
x_{i} \\
x_{j}
\end{array}\right\}=\left[\begin{array}{ll}
\boldsymbol{H}_{i i} & \boldsymbol{H}_{i j} \\
\boldsymbol{H}_{j i} & \boldsymbol{H}_{j j}
\end{array}\right]\left\{\begin{array}{l}
f_{i} \\
f_{j}
\end{array}\right\}
$$

where $\boldsymbol{H}$ is the FRF, superscripts " 1 " and "2" denote the substructure in the assembled structure, $x_{i}$ and $x_{j}$ are external node and joint node displacement vectors of the substructure, respectively, $f_{i}$ and $f_{j}$ are force vectors applied at the external nodes of substructure and joint nodes of the assembled structure, respectively.

Similarly, the input-output relationship for substructures can be written as

$$
\left\{\begin{array}{l}
x_{a} \\
x_{b}
\end{array}\right\}=\left[\begin{array}{ll}
\boldsymbol{H}_{a a} & \boldsymbol{H}_{a b} \\
\boldsymbol{H}_{b a} & \boldsymbol{H}_{\mathrm{bb}}
\end{array}\right]\left\{\begin{array}{l}
f_{a} \\
f_{b}
\end{array}\right\}
$$

The dynamic characteristics of a joint system can be expressed as

$$
f_{c}=\boldsymbol{D}_{j} x_{c}
$$

From the mechanical equilibrium condition and displacement compatibility condition, it is obvious that

$$
\begin{gathered}
f_{b}+f_{c}=f_{j} \\
x_{b}=x_{c}=x_{j}
\end{gathered}
$$

Rearranging Eq. (2) leads to

$$
x_{b}=x_{c}=\boldsymbol{H}_{b a} f_{a}+\boldsymbol{H}_{\mathrm{bb}} f_{b}
$$

Substituting Eq. (3) into Eq. (6) yields 


$$
f_{j}-f_{b}=\boldsymbol{D}_{j} x_{c}=\boldsymbol{D}_{j} \boldsymbol{H}_{b a} f_{a}+\boldsymbol{D}_{j} \boldsymbol{H}_{b b} f_{b}
$$

From Eq. (3) Eq. (7), it is obvious that

$$
\left\{\begin{array}{c}
x_{i} \\
x_{j}
\end{array}\right\}=\left(\left[\begin{array}{c}
\boldsymbol{H}_{a a} \\
\boldsymbol{H}_{b a}
\end{array}\right]-\left[\begin{array}{c}
\boldsymbol{H}_{a b} \\
\boldsymbol{H}_{b b}
\end{array}\right]\left(\mathrm{I}+\boldsymbol{D}_{j} \boldsymbol{H}_{b b}\right)^{-1} \boldsymbol{D}_{j} \boldsymbol{H}_{b a}\right)\left\{f_{i}\right\}+\left[\begin{array}{c}
\boldsymbol{H}_{a b} \\
\boldsymbol{H}_{\mathrm{bb}}
\end{array}\right]\left(\mathrm{I}+\boldsymbol{D}_{j} \boldsymbol{H}_{b b}\right)^{-1}\left\{f_{j}\right\}
$$

Comparing Eq. (8) with Eq. (1) yields

$$
\begin{array}{r}
{\left[\begin{array}{l}
\boldsymbol{H}_{i i} \\
\boldsymbol{H}_{j i}
\end{array}\right]=\left[\begin{array}{l}
\boldsymbol{H}_{a a} \\
\boldsymbol{H}_{b a}
\end{array}\right]-\left[\begin{array}{l}
\boldsymbol{H}_{i j} \\
\boldsymbol{H}_{i j}
\end{array}\right] \boldsymbol{D}_{j} \boldsymbol{H}_{b a}} \\
{\left[\begin{array}{l}
\boldsymbol{H}_{i j} \\
\boldsymbol{H}_{i j}
\end{array}\right]=\left[\begin{array}{l}
\boldsymbol{H}_{a b} \\
\boldsymbol{H}_{b b}
\end{array}\right]\left(\mathrm{I}+\boldsymbol{D}_{j} \boldsymbol{H}_{b b}\right)^{-1}}
\end{array}
$$

Rearranging Eq. (9) and Eq. (10) leads to

$$
\begin{aligned}
& \boldsymbol{H}_{i j} \boldsymbol{D}_{j} \boldsymbol{H}_{b a}=\boldsymbol{H}_{a a}-\boldsymbol{H}_{i i} \\
& \boldsymbol{H}_{j j} \boldsymbol{D}_{j} \boldsymbol{H}_{b a}=\boldsymbol{H}_{b a}-\boldsymbol{H}_{j i} \\
& \boldsymbol{H}_{i j} \boldsymbol{D}_{j} \boldsymbol{H}_{b b}=\boldsymbol{H}_{a b}-\boldsymbol{H}_{i j} \\
& \boldsymbol{H}_{j j} \boldsymbol{D}_{j} \boldsymbol{H}_{b b}=\boldsymbol{H}_{b b}-\boldsymbol{H}_{i j}
\end{aligned}
$$
(11):

The dynamic stiffness can be obtained by using generalized inverse matrix based on Eq.

$$
\begin{gathered}
\boldsymbol{Y} \cdot \boldsymbol{T} \boldsymbol{f}(\omega)=\boldsymbol{H}_{j}^{-1}(\omega) \\
\boldsymbol{Y}=\left[\begin{array}{lll}
\boldsymbol{K} & \boldsymbol{C} & M
\end{array}\right] \\
\boldsymbol{T}_{f}(\omega)=\left[\begin{array}{lll}
\boldsymbol{I} & \boldsymbol{i} \omega \boldsymbol{I} & -\omega^{2} \boldsymbol{I}
\end{array}\right]^{T}
\end{gathered}
$$

where $\boldsymbol{Y}(\boldsymbol{\omega})$ is the coefficient matrix structured by stiffness, damper, and mass matrices, $\boldsymbol{T}_{\boldsymbol{f}}(\boldsymbol{\omega})$ is transformation matrix used to uncouple $\boldsymbol{Y}(\boldsymbol{\omega})$.

Extending Eq. (12) leads to

$$
\begin{gathered}
\tilde{\boldsymbol{Y}} \cdot\left[\begin{array}{lll}
\boldsymbol{T}_{f}\left(\boldsymbol{\omega}_{1}\right) & \cdots & \boldsymbol{T}_{f}\left(\boldsymbol{\omega}_{1}\right)
\end{array}\right]=\left[\begin{array}{lll}
\boldsymbol{H}_{j}^{-1}\left(\boldsymbol{\omega}_{1}\right) & \cdots & \boldsymbol{H}_{j}^{-1}\left(\boldsymbol{\omega}_{n}\right)
\end{array}\right] \\
\tilde{\boldsymbol{Y}}=\left[\begin{array}{llll}
\boldsymbol{H}_{j}^{-1}\left(\omega_{1}\right) & \cdots & \boldsymbol{H}_{j}^{-1}\left(\omega_{n}\right)
\end{array}\right] \cdot\left[\begin{array}{lll}
\boldsymbol{T}_{f}\left(\omega_{1}\right) & \cdots & \boldsymbol{T}_{f}\left(\omega_{1}\right)
\end{array}\right]^{+} \\
\tilde{\boldsymbol{Y}}=\left[\begin{array}{lll}
\tilde{\boldsymbol{K}} & \tilde{\boldsymbol{C}} & \tilde{\boldsymbol{M}}
\end{array}\right]
\end{gathered}
$$

where $\tilde{\boldsymbol{Y}}$ is the result matrix structured by the equivalent stiffness, damper, and mass factor $(\tilde{\boldsymbol{K}}, \tilde{\boldsymbol{C}}$ and $\tilde{\boldsymbol{M}})$.

\section{Examples}

To validate the feasibility and accuracy of the proposed method, an experimental examples is presented here for dynamic modeling of single stiffness to prove the correctness of the method proposed in this paper. The test assembly is used in the experimental example, which is consisted of one steel beam and one cast iron beam, with a same rectangular cross section $\left(30 * 10 \mathrm{~mm}^{2}\right)$ and a length of $350 \mathrm{~mm}$. Both the beams are suspended by two flexible springs during testing. The responses of each structure are obtained by applying an impact force on the input point with a hammer whose response being obtained at the output points with an accelerometer. All the data are processed by LMS test lab, and transmitted to computer for further calculation. A bolted joint with a torque of $60 \mathrm{Nm}$ applied on is used to assemble the substructures. The experimental set-up and distribution of test points are shown in figure 2, the picture of field test is shown in figure 3, and the material data of beams is shown in table 1 . 

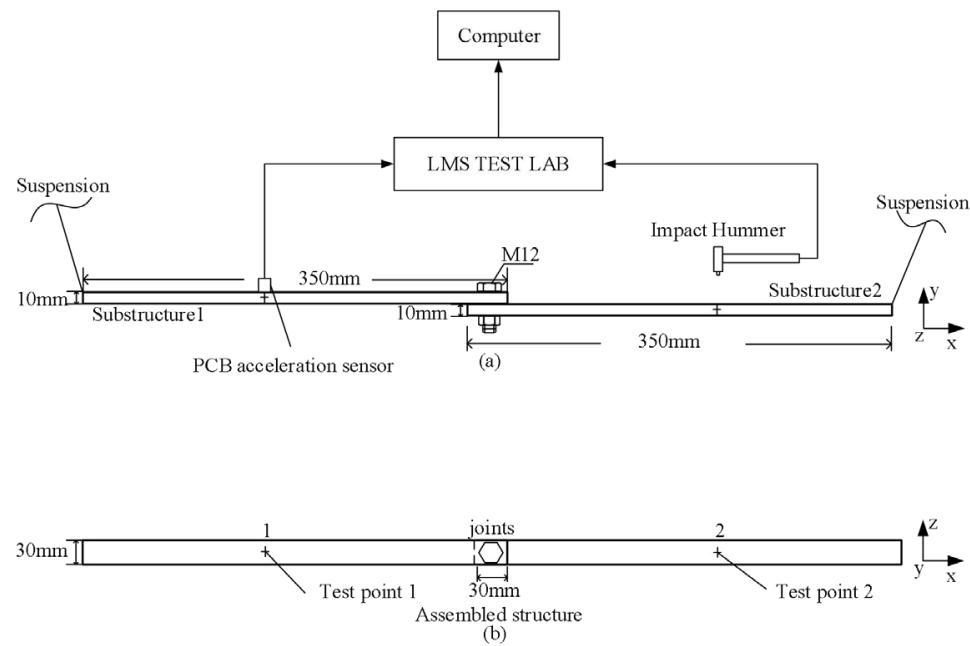

Fig. 2. (a) Experimental set-up for impact hammer test; (b) Distribution of test point.

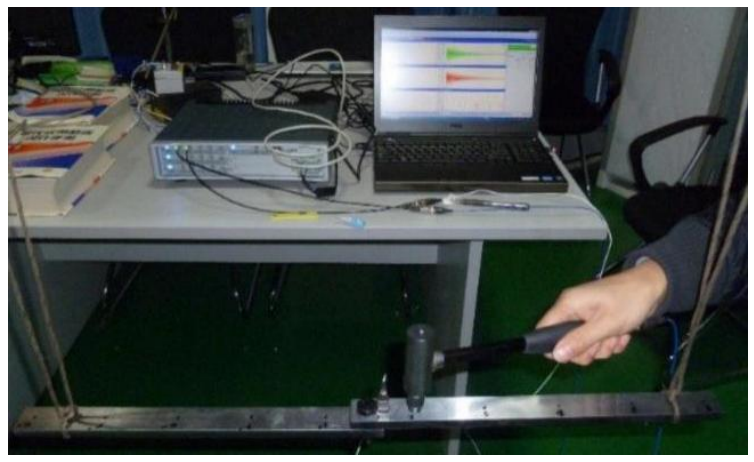

Fig. 3. Picture of field test.

Table 1. Material data of beams.

\begin{tabular}{|c|c|c|}
\hline & Substructure 1 & Substructure 2 \\
\hline Material & Steel & Cast iron \\
\hline Young's modulus $\left(\mathrm{N} / \mathrm{m}^{2}\right)$ & $2.10 \mathrm{E} 11$ & $1.3 \mathrm{E} 11$ \\
\hline Poisson ratio & 0.3 & 0.3 \\
\hline Density $\left(\mathrm{kg} / \mathrm{m}^{3}\right)$ & 7850 & 7350 \\
\hline
\end{tabular}

The measured FRF data of substructure and assembled structure is shown in figure 4.

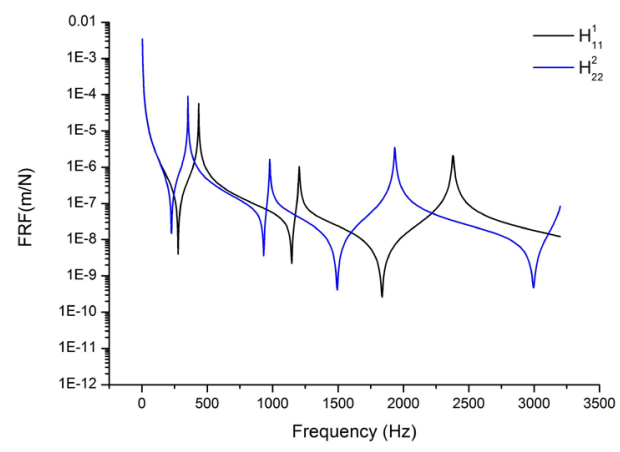

(a)

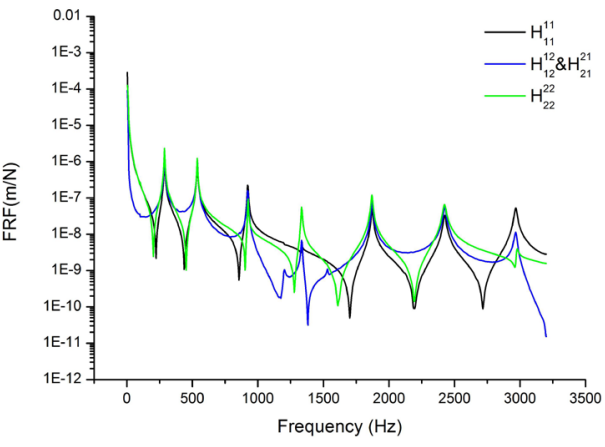

(b)

Fig. 4. (a) Part of FRF data of substructure; (b) FRF data of assembly structure. 
The FEA model is built, which is shown in figure 5. The comparison between the measured value and the predicted value is shown in figure 6 .

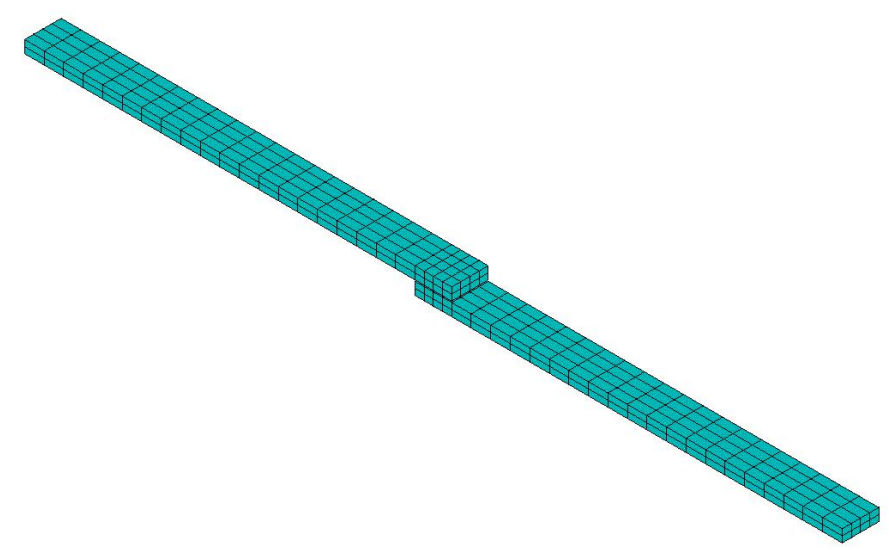

Fig. 5. Finite element model of assembly system.

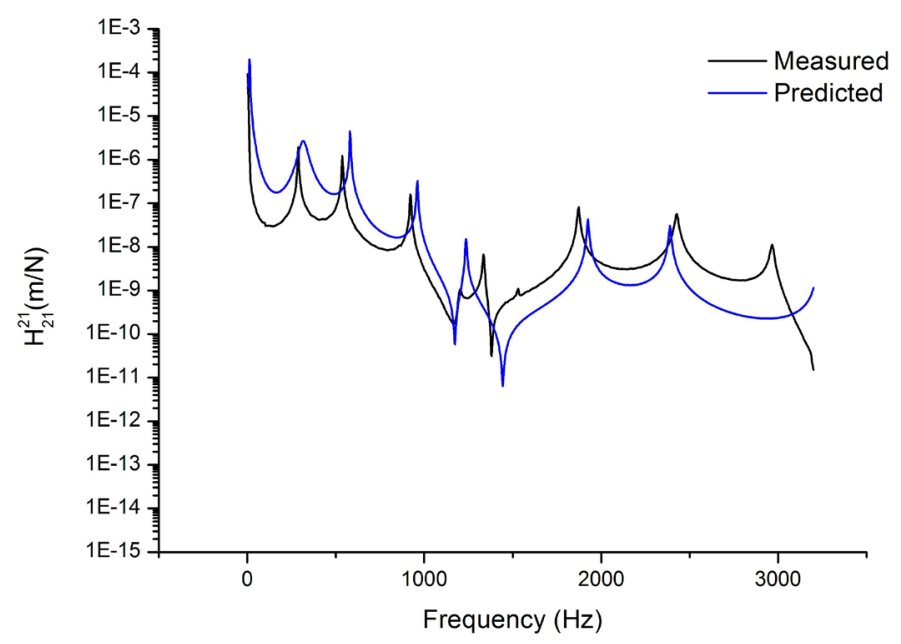

Fig. 6. Comparison between the measured value and the predicted value.

\section{Conclusion}

In this paper, the formulas for identifying the joints dynamic properties are deduced based on the mechanical equilibrium condition and the displacement compatibility condition. The theoretical model is built based on FRFs, and the equivalent dynamic stiffness is obtained by using least square method. One experimental example is provided to validate the feasibility and the accuracy of the proposed method, showing good fitting with experimental results.

\section{Conflict of Interests}

The authors declare that there is no conflict of interests regarding the publication of this paper. 


\section{Acknowledgment}

This work is sponsored by National Science and Technology Major Project (Grant no. 2013ZX04005-012).

\section{References}

1. Beards, C. F. (1983). The damping of structural vibration by controlled interfacial slip in joints. Journal of Vibration \& Acoustics, 105(3), 369-373.

2. Ren Y, Beards CF. On the importance of weighting for FRF joint identification. In: The 11th International Modal Analysis Conference, Florida, USA, 1993.

3. Okubo, N., \& Miyazaki, M. (1984). Development of uncoupling technique and its application. In Proceedings of International Modal Analysis Conference, Florida, USA (pp. 1194-1200).

4. Tsai, J. S., \& Chou, Y. F. (1988). The identification of dynamic characteristics of a single bolt joint. Journal of Sound \& Vibration, 125(3), 487-502.

5. Ren, Y., \& Cf., B. (1995). Identification of joint properties of a structure using frf data. Journal of Sound Vibration, 186(4), 567-587.

6. Liu, \& Wen jie. (2001). Structural dynamic analysis and testing of coupled structures. Imperial College London.

7. Yang, T., Fan, S. H., \& Lin, C. S. (2003). Joint stiffness identification using frf measurements. Computers \& Structures, 81(s 28-29), 2549-2556.

8. Čelič, D., \& Boltežar, M. (2009). The influence of the coordinate reduction on the identification of the joint dynamic properties. Mechanical Systems \& Signal Processing, 23(4), 1260-1271. 